Recepción: 15 / 04 / 2017

Ciencias Médicas

Aceptación: 01 / 05 / 2017

Articulo Científico

Publicación: 15 / 05 / 2017

\title{
Financiamiento del programa de maternidad gratuita en un centro de salud
}

\author{
Financing of the free maternity program in a health center
}

\section{Financiamento programa de maternidade livre em um centro de saúde}

\author{
Grace V. Bayas- Huilcapi \\ gbayas@utb.edu.ec
}

Silvana M. Heredia-Flores " silvanita_166@hotmail.com

Martha B. Baquerizo-Cabrera martha.baquerizoc@ug.edu.ec

Correspondencia: gbayas@utb.edu.ec

\footnotetext{
Magister en Gerencia de Servicios de Salud; Diploma Superior en Atención Primaria en Salud; Especialista en Gestión de Servicios de Salud; Diploma Superior en Gestión de Desarrollo de los Servicios de Salud; Doctora en Medicina y Cirugía; Docente Universidad Técnica de Babahoyo, Ecuador.

II. Magister en Gerencia Educativa; Especialista en Gestión de Procesos Educativos; Diplomado en Docencia Superior; Doctora en Medicina y Cirugía; Universidad Técnica de Babahoyo, Ecuador.

III. Magister en Gerencia Educativa; Especialista en Gestión de Procesos Educativos; Diplomado en Docencia Superior; Doctora en Medicina y Cirugía; Universidad de Guayaquil, Ecuador.
} 


\title{
Resumen
}

Siendo el Programa de Maternidad Gratuita uno de los programas de mayor trascendencia en el sector salud, ya que involucra uno de los grupos más vulnerables de la población, como son las mujeres en edad fértil, embarazadas y niños hasta los 5 años de edad; en ningún momento debería presentar ningún tipo de falencia, más aun si el Gobierno central presenta una promoción con una satisfacción del $100 \%$ de sus usuarios tanto internos como externos.

\begin{abstract}
Al ser el Ecuador uno de los países con uno de los más altos índices de embarazos en adolescentes y desnutrición infantil, estaría de más querer demostrar que la correcta aplicación de dicho programa ayudaría de una manera importante y trascendental a corregir o disminuir de manera considerable dichas tasas negativas.
\end{abstract}

Palabras clave: Financiamiento; programa de maternidad; centro de salud. 


\begin{abstract}
The Free Maternity Program is one of the most important programs in the health sector, since it involves one of the most vulnerable groups of the population, such as women of childbearing age, pregnant women and children up to 5 years of age; At no time should it present any type of bankruptcy, even more so if the central government presents a promotion with $100 \%$ satisfaction of its users both internal and external.
\end{abstract}

Ecuador being one of the countries with one of the highest rates of adolescent pregnancies and child undernutrition, it would be more desirable to demonstrate that the correct application of said program would help in an important and transcendental way to correct or significantly reduce said Negative rates.

Key words: Financing; maternity program; clinic. 


\section{Resumo}

Sendo o Programa Livre Maternidade um dos programas mais significativos no sector da saúde, uma vez que envolve um dos grupos mais vulneráveis da população, como as mulheres em idade fértil, gestantes e crianças até aos 5 anos de idade; em nenhum momento deve apresentar qualquer tipo de falência, ainda mais se o governo central tem uma promoção com uma satisfação de $100 \%$ de seus usuários internos e externos.

Como o Equador um país com uma das maiores taxas de gravidez na adolescência e desnutrição infantil, seria mais quero mostrar que a boa execução do programa de ajuda de uma forma ambiciosa e importante para corrigir ou reduzir consideravelmente estes taxas negativas.

Palavras chave: financiamento; programa de maternidade; centro de saúde. 


\section{Introducción.}

El principal problema de todo "programa sin fines de lucro" es la politización de quienes tienen que ver con la dirección de los mismos, ya que la gran mayoría de veces no se toma en cuenta el objetivo para lo cual fue creado dicho programa poniendo de por medio beneficios personales o de ciertos grupos, con lo cual se pone en tela de juicio a quienes hacen el frente de batalla propiamente dicho - personal médico - ante la población, con la consiguiente ejecución inadecuada y/o incompleta de un programa de vital importancia, como es el Programa de Maternidad Gratuita, ya que dicho programa abarca aproximadamente el $60 \%$ de la población que acude al Centro de Salud Hospital Baba; y que por razones aún desconocidas dicho programa no se ha podido ejecutar en su totalidad durante ciertos periodos del año. (Lucio, Villacrés, \& Henríquez, 2011) (Instituto Nacional de Estadística y Censos, 2009)

El Programa de Maternidad Gratuita nació con el objetivo de garantizar una "maternidad saludable", Programa que compromete una buena asesoría en lo que respecta a salud sexual y reproductiva a la mujer en edad fértil, consulta pre concepcional y prenatal y a la vez la atención del producto del embarazo hasta los cinco años de edad, pero por diversos motivos no se puede brindar la atención oportuna a los usuarios de este programa, ya sea por falta de recurso humano, por falta de insumos y/o medicamentos, falta de equipamiento o equipamiento inadecuado y en gran número de ocasiones por la cultura de los usuarios. (Ortale, 2009) (Vásquez Galán , 2001)

La presente investigación incluye la revisión de los parámetros en lo que respecta a costopaciente ya sea por atención medica y/o exámenes, población atendida durante un periodo determinado -2010- y el presupuesto enviado por el ministerio de salud para cubrir dicho 
financiamiento en su totalidad. Además se debe tomar en cuenta parámetros de mucha importancia como son la satisfacción tanto del usuario interno como del usuario externo.

Además están definidos aspectos importantes que encontraremos en la situación problemática y en los antecedentes de investigaciones anteriores que son experiencias en otras instituciones de salud del sector público local y nacional, presentamos nuestros objetivos específicos, al igual que las hipótesis específicas verificadas por medio de las variables de estudio, presentamos además una recopilación bastante interesante de definiciones de términos que nos permitirán comprender en forma adecuada el proceso a implementarse y los aspectos principales sobre lo que gira la presente investigación la misma que se fundamenta en los resultados de la aplicación del instrumento cuestionario-encuesta, sus resultados nos permiten señalar la descripción en cuadros y gráficos, para luego interpretarlos y presentar nuestras conclusiones y recomendaciones sobre la que elaboramos nuestra propuesta final.

Una vez realizados los estudios necesarios, habremos establecidos los eslabones más débiles en los cuales se presentan los obstáculos en la ejecución del Programa de Maternidad Gratuita en el Centro de Salud Hospital Baba, ya sea a nivel de directivos centrales o locales, ejecutores y beneficiarios del mismo; de tal manera que dejaremos constancia a través de sugerencias, para que de una u otra manera se pueda ejecutar dicho programa con el mayor acierto posible. (Stern, 2009) (a Integrantes de la Subcomisión de Ética Clínica, 2010)

El presente estudio tiene como objetivo Establecer una propuesta que permita la optimización de los recursos: humanos, materiales y financieros para el programa de maternidad gratuita en el Centro de Salud Hospital de Baba. 


\section{Marco contextual.}

Los Ríos, Provincia de la costa Ecuatoriana, se encuentra ubicada en la cuenca hidrográfica del rió Guayas, en el centro - sur - oeste de la República del Ecuador. Es la única provincia de la región que no tiene acceso al mar. Cuenta con una extensión de 7.177,62 Km2, y, tiene una población de 650178 habitantes, de los cuales según el género corresponden a 335279 hombres y 314899 mujeres ${ }^{1}$. Políticamente se encuentra dividida en trece cantones, siendo su capital, la ciudad de Babahoyo, el cantón Baba, posee un total de 42.138 habitantes, su población urbana es de 5810 y la rural 36.328 de habitantes ${ }^{2}$.

En el contexto social el Cantón Baba es una de las poblaciones Riosenses más antiguas e inicialmente se componía de las parroquias Baba, Guare y Juana de oro, para posteriormente quedarse con Baba, Guare e Isla de Bejucal; y los recintos: La Carmela, Tinoco, Progreso, Santa Isabel, Concepción, Chínate, La Jagua, El Resbalón, Versalles, El Porvenir, San José, San Antonio, San Francisco, Campo Alegre, El Chorrón, Cimarrón, Rosa de Oro y otros. Baba es cuna de ilustres personalidades, en este lugar nació la Beata Mercedes de Jesús Molina en el recinto Guayabo, y el Dr. Francisco Xavier Aguirre Abad.

El suelo es plano en su mayoría, se encuentra a 12 metros sobre el nivel del mar. El lugar es caluroso en el invierno, pero muy fresco en el verano, su temperatura va de 26 grados a 35 grados, tiene varios ríos entre ellos, el río Arenal, el Junquillo, entre otros.

\footnotetext{
${ }^{1}$ INEC, 2004. Último Censo de Población y Vivienda. Los Ríos. S/Ed. Quito.

${ }^{2}$ IDEM
} 


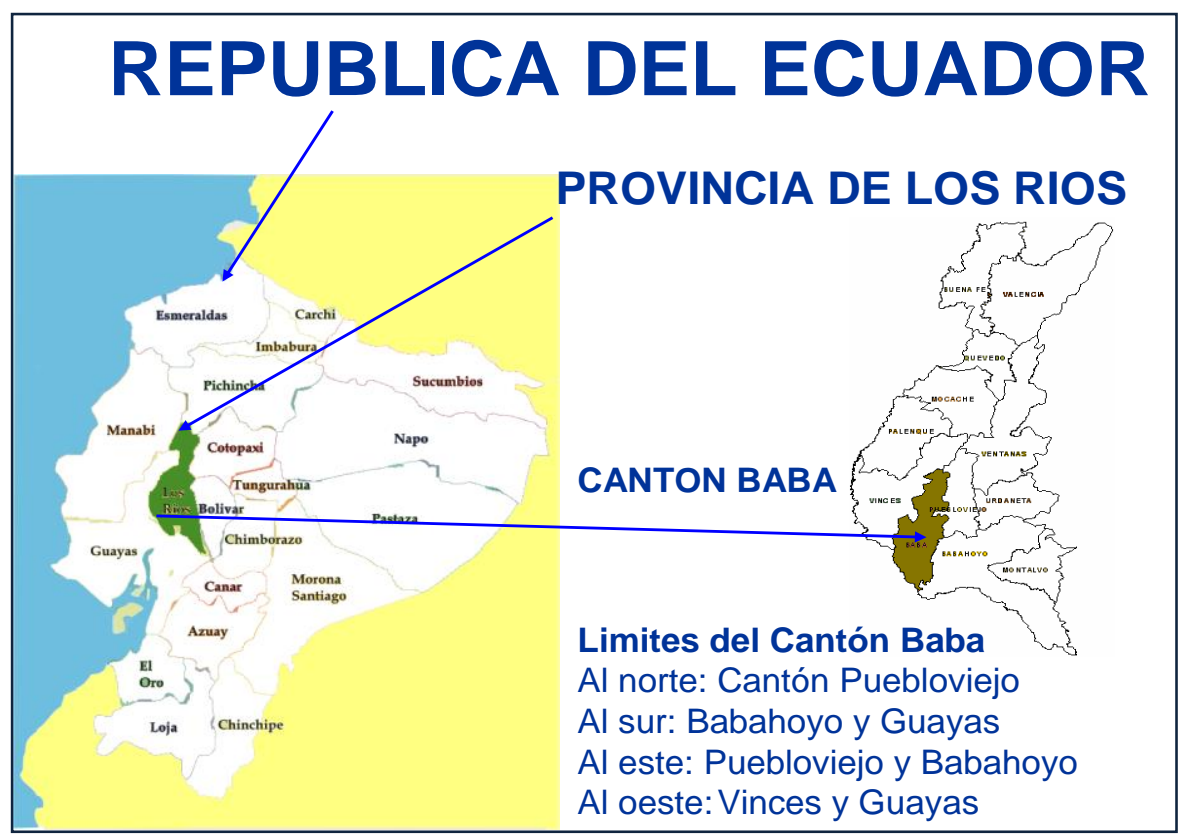

La Ciudad de Baba tiene una parroquia urbana: Baba. El cantón Baba cuenta con las siguientes parroquias rurales: Guare e Isla de Bejucal. Baba tiene una población de 42.138 habitantes, aproximadamente el 5.75\% de la población total de La Provincia de Los Ríos. Su extensión territorial es de $330 \mathrm{Km}$.

Las características de este sector en cuanto a la infraestructura de servicios básicos son deficientes. La cabecera cantonal tiene un sistema de alcantarillado tanto pluvial como sanitario obsoleto que apenas llega al 35\% de población y en las cabeceras parroquiales de Guare e Isla de Bejucal no existen. El agua de consumo humano no es potable y las 3 parroquias tienen problema de desabastecimiento.

Sumado a ello, el mal manejo de la cadena alimenticia junto con los cambios climáticos propios del medio dan lugar a la propagación de estados de malnutrición así como también a la presencia de enfermedades infectocontagiosas e incluso pudiendo presentarse como brotes epidémicos. 
El Centro de Salud Hospital Baba es el referente del Ministerio de Salud Pública en el Cantón Baba, por lo cual está en la obligación de conocer su área de influencia para de esta manera poder brindar un servicio de calidad. La población que acude a dicho Centro de Salud Hospital es aquella que está asentada en la cabecera cantonal, pero la gran mayoría está conformada por los habitantes de las áreas de influencia siendo esta de un quintil de pobreza bien marcado y con un nivel sociocultural muy bajo. Además de aquello, es punto de referencia de otros centros de salud, como los de las parroquias - Guare, Isla de Bejucal, La Carmela - quienes forman parte de la misma área de salud $\mathrm{N}^{\circ}$. 6, o de sub-centros de otras áreas de salud, que por razones geográficas $-\mathrm{La}$ Carolina, Pimocha- les es mucho más fácil el acceso al Centro de Salud Hospital Baba.

A pesar de lo antes expuesto y siendo el Cantón Baba uno de los cantones más antiguos del Ecuador, recién hace aproximadamente 25 años; se crea el Centro de Salud Hospital Baba asentándose en la Avenida Quito y Urdaneta, para brindar atención de salud en dicha población, el cual se inició como un Hospital Básico ya que brindaba los servicios de medicina general, cirugía general, gineco-obstetricia, pediatría, especialidades las cuales eran cubiertas por médicos generales y no por especialistas. Además cuenta con la atención del servicio de laboratorio clínico, servicio de rayos $\mathrm{x}$, inspectoría de salud, servicio de odontología, servicios que se consideró adecuados para la población de ese entonces ya que contaba con el personal y espacio físico necesario para satisfacer las necesidades en lo que a salud se refiere, además se crearon las partidas presupuestarias necesarias para llenar dichos cargos. 


\section{Resultados.}

\section{Pruebas Estadísticas aplicadas en la verificación de las hipótesis}

Las pruebas estadísticas aplicadas en el presente estudio corresponden a:

Encuestas: aplicadas a usuarios internos y externos.

Entrevistas: aplicadas a directivos y personal estadístico.

\section{Análisis e Interpretación de datos}

\section{Pregunta $\mathbf{N}^{\circ} 1$}

¿Existe una proyección adecuada del crecimiento poblacional de los beneficiarios del programa de maternidad gratuita en el CSH Baba?

\section{Cuadro $\mathbf{N}^{\circ} 1$}

\begin{tabular}{|c|c|c|c|c|c|}
\hline SI & $\%$ SI & NO & $\%$ NO & NUMERO & INDICE \\
\hline 22 & $48,9 \%$ & 23 & $51,1 \%$ & 45 & $100 \%$ \\
\hline
\end{tabular}

El 51\% de usuarios internos contestó que no existe una proyección adecuada del crecimiento poblacional de los beneficiarios del programa de maternidad gratuita en el Centro de Salud Hospital Baba 


\section{Pregunta $\mathrm{N}^{\circ} 2$}

¿Existen los insumos necesarios para la aplicación del Programa de Maternidad Gratuita en el Centro de Salud Hospital Baba?

\section{Cuadro $\mathbf{N}^{\circ} 2$}

\begin{tabular}{|c|c|c|c|c|c|}
\hline SI & $\%$ SI & NO & $\%$ NO & NUMERO & INDICE \\
\hline 13 & $28,9 \%$ & 32 & $71,1 \%$ & 45 & $100 \%$ \\
\hline
\end{tabular}

El $71 \%$ de usuarios internos contestó que no existen los insumos necesarios para la aplicación del Programa de Maternidad Gratuita en el Centro de Salud Hospital Baba.

\section{Pregunta $\mathbf{N}^{\circ} 3$}

¿Existen las condiciones adecuadas para la aplicación del Programa de Maternidad Gratuita?

\begin{tabular}{|c|c|c|c|c|c|}
\hline SI & \% SI & NO & $\%$ NO & NUMERO & INDICE \\
\hline 10 & $22,2 \%$ & 35 & $77,8 \%$ & 45 & $100 \%$ \\
\hline
\end{tabular}

El 78\% de usuarios internos contestó que no existen las condiciones adecuadas para la aplicación del Programa de Maternidad Gratuita. 


\section{Pregunta $N^{\circ} 4$}

¿Existe el recurso humano suficiente para la aplicación del Programa de Maternidad Gratuita en el CSH Baba?

\section{Cuadro $\mathbf{N}^{\circ} 4$}

\begin{tabular}{|c|c|c|c|c|c|}
\hline SI & $\%$ SI & NO & \% NO & NUMERO & INDICE \\
\hline 07 & $15,6 \%$ & 38 & $84,4 \%$ & 45 & $100 \%$ \\
\hline
\end{tabular}

El $84 \%$ de usuarios internos contestó que no existe el recurso humano suficiente para la aplicación del Programa de Maternidad Gratuita en el Centro de Salud Hospital Baba.

\section{Pregunta $\mathbf{N}^{\circ} 5$}

¿Considera usted que el Gobierno central envía los recursos necesarios para la aplicación del Programa de Maternidad Gratuita en el CSH Baba?

\section{Cuadro $\mathbf{N}^{\circ} 5$}

\begin{tabular}{|c|c|c|c|c|c|}
\hline SI & $\%$ SI & NO & $\%$ NO & NUMERO & INDICE \\
\hline 05 & $11,1 \%$ & 40 & $88,9 \%$ & 45 & $100 \%$ \\
\hline
\end{tabular}


El $89 \%$ de usuarios internos contestó que consideran que el Gobierno central no envía los recursos necesarios para la aplicación del Programa de Maternidad Gratuita en el CSH Baba.

\section{Pregunta $\mathbf{N}^{\circ} 6$}

¿Considera usted que el Programa de Maternidad Gratuita se aplica correctamente en el CSH Baba?

\section{Cuadro $\mathbf{N}^{\circ} 6$}

\begin{tabular}{|c|c|c|c|c|c|}
\hline SI & $\%$ SI & NO & $\%$ NO & NUMERO & INDICE \\
\hline 08 & $17,8 \%$ & 37 & $82,2 \%$ & 45 & $100 \%$ \\
\hline
\end{tabular}

El $82 \%$ de usuarios internos contestó que consideran que el Programa de Maternidad Gratuita no se aplica correctamente en el CSH Baba.

\section{Pregunta $\mathbf{N}^{\circ} 7$}

¿Conoce sobre el Programa de Maternidad Gratuita?

\section{Cuadro $\mathbf{N}^{\circ} 7$}

\begin{tabular}{|c|c|c|c|c|c|}
\hline SI & $\%$ SI & NO & $\%$ NO & NUMERO & INDICE \\
\hline 355 & $93,7 \%$ & 24 & $6,3 \%$ & 379 & $100 \%$ \\
\hline
\end{tabular}

El 94\% de usuarios externos contestó que si conoce el Programa de Maternidad Gratuita. 


\section{Pregunta $\mathbf{N}^{\circ} 8$}

¿Usted cree que existe personal calificado - especialistas - para la aplicación del Programa de Maternidad Gratuita en el Centro de Salud Hospital Baba?

\section{Cuadro $\mathbf{N}^{\circ} 8$}

\begin{tabular}{|c|c|c|c|c|c|}
\hline SI & \% SI & NO & \% NO & NUMERO & INDICE \\
\hline 361 & $95,2 \%$ & 18 & $4,8 \%$ & 379 & $100 \%$ \\
\hline
\end{tabular}

El 95\% de usuarios externos contestó que si cree que existe personal calificado especialistas - para la aplicación del Programa de Maternidad Gratuita en el Centro de Salud Hospital Baba

\section{Pregunta $\mathbf{N}^{\circ} 9$}

¿Usted cree que el Programa de Maternidad Gratuita cuenta con insumos - medicinas - necesarios?

\section{Cuadro $\mathbf{N}^{\circ} 9$}

\begin{tabular}{|c|c|c|c|c|c|}
\hline SI & \% SI & NO & \% NO & NUMERO & INDICE \\
\hline 279 & $73,6 \%$ & 100 & $26,4 \%$ & 379 & $100 \%$ \\
\hline
\end{tabular}


El 74\% de usuarios externos contestó que si cree que el Programa de Maternidad Gratuita cuenta con insumos - medicinas - necesarios.

\section{Pregunta $\mathbf{N}^{\circ} 10$}

¿Usted cree que el Centro de Salud Hospital Baba posee la infraestructura necesaria para la aplicación del Programa de Maternidad Gratuita?

\section{Cuadro $\mathbf{N}^{\circ} \mathbf{1 0}$}

\begin{tabular}{|c|c|c|c|c|c|}
\hline SI & $\%$ SI & NO & $\%$ NO & NUMERO & INDICE \\
\hline 313 & $82,6 \%$ & 66 & $17,4 \%$ & 379 & $100 \%$ \\
\hline
\end{tabular}

El $83 \%$ de usuarios externos contestó que si cree que el Centro de Salud Hospital Baba posee la infraestructura necesaria para la aplicación del Programa de Maternidad Gratuita.

\section{Pregunta $N^{\circ} 11$}

¿Acude regularmente a los controles en el Centro de Salud Hospital Baba?

\section{Cuadro $\mathbf{N}^{\circ} 11$}

\begin{tabular}{|c|c|c|c|c|c|}
\hline SI & $\%$ SI & NO & \% NO & NUMERO & INDICE \\
\hline 368 & $97,1 \%$ & 11 & $2,9 \%$ & 379 & $100 \%$ \\
\hline
\end{tabular}


El 97\% de usuarios externos contestó que si acude regularmente a los controles en el Centro de Salud Hospital Baba.

\section{Pregunta $\mathbf{N}^{\circ} 12$}

¿Cree usted que el Programa de Maternidad Gratuita del Centro de Salud Hospital Baba cubre satisfactoriamente las necesidades de salud de los grupos incluidos en el mismo?

\section{Cuadro $\mathbf{N}^{\circ} 12$}

\begin{tabular}{|c|c|c|c|c|c|}
\hline SI & \% SI & NO & \% NO & NUMERO & INDICE \\
\hline 301 & $79,4 \%$ & 78 & $20,6 \%$ & 379 & $100 \%$ \\
\hline
\end{tabular}

El 79\% de usuarios externos contestó que si cree que el Programa de Maternidad Gratuita del Centro de Salud Hospital Baba cubre satisfactoriamente las necesidades de salud de los grupos incluidos en el mismo.

\section{Conclusiones.}

De las encuestas y entrevistas realizadas se puede concluir que al no haber una proyección adecuada del crecimiento poblacional por parte del departamento de estadística para los beneficiarios del Programa de Maternidad Gratuita, es totalmente imposible determinar el incremento de usuarios externos que se benefician de dicho programa.

En la entrevista realizada a directivos se concluye que no se ha creado proyectos para corregir las falencias. No hay normativas para asegurar la proyección del crecimiento poblacional. 
Por tal motivo se presenta una propuesta en la cual se corrijan todos los factores de tipo estadístico, financiero y de aplicación del Programa de Maternidad Gratuita.

Sumado a lo anteriormente dicho no existen parámetros reales de los beneficiarios del Programa de Maternidad Gratuita de años anteriores con la consiguiente reposición incompleta de los recursos utilizados para la aplicación del programa.

Los usuarios externos como consumidores finales de una u otra forma reciben los beneficios del programa de maternidad gratuita, aunque de manera incompleta pero siendo este servicio gratuito, los usuarios se sienten "satisfechos" a pesar de que este no los cubra al ciento por ciento en su totalidad, pero mucho más importante que una pseudo-satisfacción es el hecho mismo de que el Programa de Maternidad Gratuita culmine con una maternidad y niños menores de 5 años saludables.

De tal manera se establece la integración de un buen sistema operativo y/o de estadística que nos permita un excelente flujo de todos los beneficiarios de dicho programa con las respectivas proyecciones de crecimiento poblacional de los mismos. Con lo cual se exige los recursos necesarios para la población real que es beneficiaria de dicho programa con lo cual también se evita una reposición incompleta de los recursos utilizados el año anterior. Inicialmente esto va a crear una situación de controversia a nivel de los organismos centrales ya que en ningún momento se estuvo atendiendo una población real lo cual siempre ha dado como resultado una demanda insatisfecha.

\section{Bibliografía.}

Integrantes de la Subcomisión de Ética Clínica. (2010). Embarazos en niñas y adolescentes. Arch Argent Pediatr, 108(6), 562-565.

Instituto Nacional de Estadística y Censos. (2009). Indicadores básicos de salud. Ecuador 2009. Quito: INEC. 
Lucio, R., Villacrés, N., \& Henríquez, R. (2011). Sistema de salud de Ecuador. Salud Pública de México, 53(2), S177-S187.

Ortale, S. (2009). Programas de salud sexual y reproductiva y maternidad adolescente en La Plata (Buenos Aires, Argentina). Avá, 15(dic), 271-288.

Stern, C. (2009). Vulnerabilidad social y embarazo adolescente en México. Papeles de POBLACIÓN, 39(1), 129-158.

Vásquez Galán , B. (2001). Salud y maternidad en la frontera México-Estados Unidos. El caso de la región Matamoros-Brownsville. Frontera Norte, 13(2), 243-281. 